\title{
Prospective investigation of risk factors for prostate cancer in the UK Biobank cohort study
}

\begin{abstract}
Aurora Perez-Cornago ${ }^{*},{ }^{\text {, Timothy } \mathrm{J} \mathrm{Key}}{ }^{1}$, Naomi E Allen ${ }^{2}$, Georgina K Fensom ${ }^{1}$, Kathryn E Bradbury ${ }^{1}$, Richard M Martin ${ }^{3,4,5}$ and Ruth C Travis ${ }^{1}$

${ }^{1}$ Cancer Epidemiology Unit, Nuffield Department of Population Health, University of Oxford, Richard Doll Building, Roosevelt Drive, Oxford OX3 7LF, UK; ${ }^{2}$ Clinical Trial Service Unit and Epidemiological Studies Unit, Nuffield Department of Population Health, Big Data Institute, University of Oxford, Oxford OX3 7LF, UK; ${ }^{3}$ School of Social and Community Medicine, University of Bristol, 39 Whatley Road, Bristol BS6 7QD, UK; ${ }^{4}$ Medical Research Council/University of Bristol Integrative Epidemiology Unit, University of Bristol, Oakfield House, Oakfield Grove, Bristol BS8 2BN, UK and ${ }^{5}$ National Institute for Health Research Bristol Biomedical Research Unit in Nutrition, Bristol Education \& Research Centre, Upper Maudlin Street, Bristol BS2 8AE, UK
\end{abstract}

Background: Prostate cancer is the most common cancer in British men but its aetiology is not well understood. We aimed to identify risk factors for prostate cancer in British males.

Methods: We studied 219335 men from the UK Biobank study who were free from cancer at baseline. Exposure data were collected at recruitment. Prostate cancer risk by the different exposures was estimated using multivariable-adjusted Cox proportional hazards models.

Results: In all, 4575 incident cases of prostate cancer occurred during 5.6 years of follow-up. Prostate cancer risk was positively associated with the following: black ethnicity (hazard ratio black vs white $=2.61,95 \%$ confidence interval =2.10-3.24); having ever had a prostate-specific antigen test (1.31, 1.23-1.40); being diagnosed with an enlarged prostate (1.54, 1.38-1.71); and having a family history of prostate cancer $(1.94,1.77-2.13$ ). Conversely, Asian ethnicity (Asian vs white hazard ratio $=0.62,0.47-0.83$ ), excess adiposity (body mass index ( $\geqslant 35$ vs $<25 \mathrm{~kg} \mathrm{~m}^{-2}=0.75,0.64-0.88$ ) and body fat ( $\geqslant 30.1$ vs $<20.5 \%=0.81,0.73-0.89$ )), cigarette smoking (current vs never smokers $=0.85,0.77-0.95$ ), having diabetes $(0.70,0.62-0.80$ ), and never having had children $(0.89,0.81-0.97)$ or sexual intercourse $(0.53,0.33-0.84)$ were related to a lower risk.

Conclusions: In this new large British prospective study, we identified associations with already-established, putative and possible novel risk factors for being diagnosed with prostate cancer. Future research will examine associations by tumour characteristics.

Prostate cancer is the most common cancer in men in the United Kingdom (UK), with 40300 cases diagnosed in 2015 (ONS, 2017). The aetiology of prostate cancer is not well understood, and the well-established risk factors age, ethnicity, and family history of the disease are not modifiable (Cuzick et al, 2014; WCRF/AICR, 2014). However, there is evidence that circulating insulin-like growth factor-I (IGF-I), which is influenced by environmental factors, is related to higher prostate cancer risk (Travis et al, 2016), and the latest World Cancer Research Fund meta-analysis of prospective studies concluded that it is probable that obesity is associated with a higher incidence of aggressive prostate cancer. While there is limited epidemiological evidence for other lifestyle risk factors for prostate cancer (WCRF/AICR, 2014), the relatively high variation in incidence rates worldwide suggests that differences in exposure to environmental factors may have a role in prostate cancer development, although some of the variation is due to differences 
between countries in prostate-specific antigen (PSA) testing (Ferlay et al, 2015).

The UK Biobank cohort is an important new resource for the study of cancer aetiology. We report here the first results from this cohort on the association between prostate cancer incidence and potential risk factors, including socio-demographic, anthropometric and lifestyle factors, health status, prostate-specific factors prior to the recruitment, sexual history, early life characteristics, hair colour, and balding pattern. We also tested whether these associations vary by time to diagnosis.

\section{MATERIALS AND METHODS}

Study design. The UK Biobank is a prospective study designed to be a resource for research into the causes of disease in middle and old age. The study protocol and information about data access are available online (http://www.ukbiobank.ac.uk/wp-content/uploads/ 2011/11/UK-Biobank-Protocol.pdf) and more details of the recruitment and study design have been published elsewhere (Sudlow et al, 2015). In brief, all participants were registered with the UK National Health Service (NHS) and lived within $\sim 25$ miles $(40 \mathrm{~km})$ of one of the assessment centres. The UK Biobank invited $\sim 9.2$ million people to participate through postal invitation with a telephone follow-up, with a response rate of $5.7 \%$. A total of 503317 men and women aged 40-69 years were recruited in 22 assessment centres across England, Wales and Scotland, between 2006 and 2010. In all, 608 participants have subsequently withdrawn from the study and their data were not available for analysis. The UK Biobank study was approved by the North West Multi-Centre Research Ethics Committee (reference number 06/ MRE08/65), and at recruitment all participants gave informed consent to participate in UK Biobank and be followed-up, using a signature capture device.

After excluding 9835 men with prevalent cancer (except C44: non-melanoma skin cancer), and 2 men censored on entry day, these analyses included a total of 219335 men (Supplementary Figure 1).

Exposure assessment. Participants provided detailed self-reported data via a touch screen questionnaire and a verbal interview with a trained nurse at the assessment centres at baseline (Sudlow et al, 2015), and a wide range of physical measurements (e.g., body mass index (BMI) and including bioimpedance) and biological samples were collected (Sudlow et al, 2015). Information about the assessment procedure is available at http://www.ukbiobank.ac.uk/.

Exposure data included information on socio-demographic factors (region, Townsend deprivation index, education level, ethnicity, employment, and living with a wife or partner), anthropometric measurements (standing height, weight, BMI, percentage body fat, waist and hip circumferences, waist to hip ratio (WHR) (UK-Biobank, 2014)), lifestyle characteristics (smoking status, alcohol consumption, and physical activity), healthrelated factors (vasectomy, hypertension, and diabetes), prostatespecific factors prior to recruitment (PSA test, enlarged prostate, and family history of prostate cancer), sexual history (number of children, age at first sexual intercourse, lifetime heterosexual partners, same-sex intercourse, and lifetime number of same-sex partners), early life factors (puberty as defined by age of first facial hair, relative age voice broke, and comparative body size and height at age 10 years), and hair colour and balding pattern. Detailed information regarding how these variables were collected is given in the Supplementary Methods.

Outcome assessment. Men were followed-up until the censoring date (30 September 2014 in England and Wales, and 31 December 2014 in Scotland) via record linkage to the NHS Central Register, which provides information on cancer registrations and deaths.
The end point included in these analyses is first diagnosis of prostate cancer (International Classification of Diseases Tenth revision codes: C61; (WHO, 2010)) or death from prostate cancer, whichever was first. Person-years were calculated from the date of recruitment to the date of cancer registration (first malignant neoplasm, except non-melanoma skin cancer (ICD-10 C44)), death, or the censoring date, whichever occurred first.

Statistical analysis. Cox proportional hazards models were used to calculate hazard ratios (HRs) and 95\% confidence intervals (CIs) for prostate cancer risk, using age as the underlying time variable. All analyses were stratified by geographical region of recruitment (10 UK regions, except for when region was the main exposure of interest) and age $(<45,45-49,50-54,55-59,60-64, \geqslant 65$ years $)$ at recruitment. Exposure variables (socio-demographic factors, anthropometric measurements, lifestyle characteristics, health status, prostate-specific factors prior to recruitment, sexual history, early life factors, and hair colour and balding pattern) were divided into categories based on their distribution at baseline in the whole cohort (categories for each exposure are explained in detailed in Supplementary Methods). Missing and/or unknown values of the exposure of interest were not included in the analyses, but missing and/or unknown values were assigned to a separate category when the variable was included as a covariate. As these are the first analyses on the association between potential risk factors and incidence of total prostate cancer in UK Biobank, potential confounders were first identified a priori based on possible risk factors for prostate cancer that have some support in the literature (Sutcliffe \& Colditz, 2013; Cuzick et al, 2014; WCRF/AICR, 2014; Rider et al, 2016).

Minimally adjusted Cox regression models were performed to identify statistically important covariates from the a priori potential confounders. On the basis of results from the minimally adjusted Cox regression analyses, the multivariable-adjusted model was additionally adjusted for Townsend deprivation score (fifths, unknown (0.1\%)), ethnicity (white, mixed background, Asian, black, other, and unknown $(0.7 \%)$ ), lives with a wife or partner (no, yes), BMI $\left(<25, \geqslant 25-<30, \geqslant 30-<35, \geqslant 35 \mathrm{~kg} \mathrm{~m}^{-2}\right.$, and unknown $(0.6 \%)$ ), cigarette smoking (never, former, current, and unknown $(0.7 \%)$ ), physical activity (low $(0-<10$ metabolic equivalents (METs) per week), moderate $(\geqslant 10-<50$ METs per week), high ( $\geqslant 50$ METs per week), and unknown (3.7\%)), diabetes (no, yes, and unknown (0.6\%)), enlarged prostate (no or unknown, and yes), and family history of prostate cancer (no, yes (brother or father), and unknown (45.1\%)). For each adjustment variable, missing values were assigned to a separate category. Body mass index was not included in the multivariable-adjusted model when fat mass, waist circumference, and WHR were the main exposure variables.

$P$-values from the multivariable-adjusted model for the association of each exposure with prostate cancer risk were calculated as follows: $P$-values for likelihood ratio tests for variables with more than two categories (categorical variables) were obtained comparing the model with and without the variable of interest; $P$-values for dichotomous variables were obtained comparing the reference category to the other category in the model; and $P$-values for trend were obtained using a pseudo-continuous variable equal to the median value in each category for continuous variables. The proportional hazards assumption was tested using time-varying covariates and Schoenfeld residuals and revealed no evidence of deviation from the proportional hazards assumption.

Sensitivity analyses were performed to test for heterogeneity in the associations with risk by time between recruitment and diagnosis $(<2$ and $\geqslant 2$ years $)$ to examine whether there were associations for cancers diagnosed shortly after recruitment, which could indicate reverse causality. For this purpose, we fitted 
Table 1. Baseline characteristics of all men included in the analysis and of men who developed prostate cancer

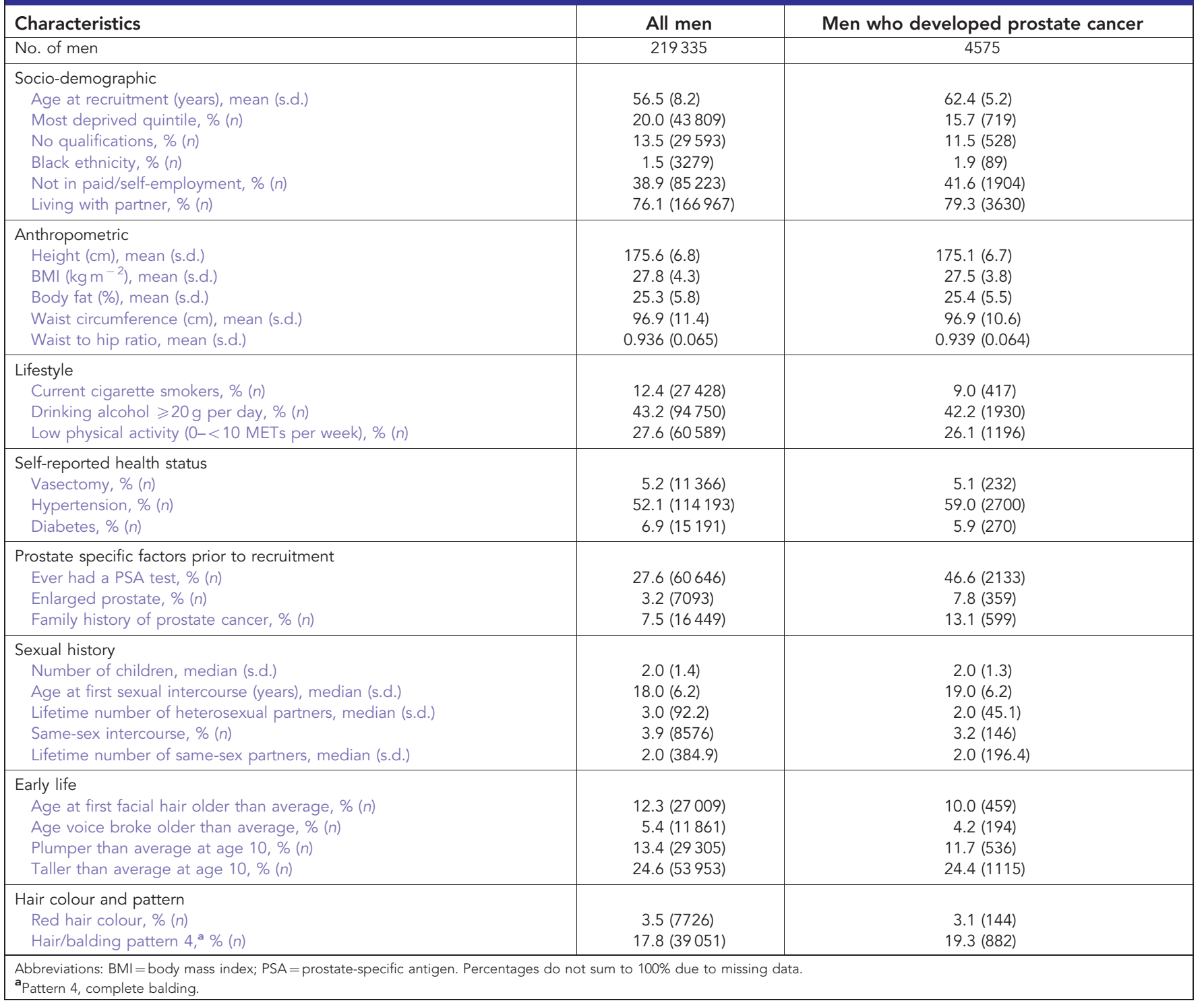

stratified Cox models based on competing risks and compared the risk coefficients and s.e.'s in the subgroups of interest $(<2$ or $\geqslant 2$ years between recruitment and diagnosis). All analyses were performed using Stata version 14.1 (Stata Corporation, College Station, TX, USA), all tests of significance were two-sided, and $P$-values $<0.05$ were considered statistically significant.

\section{RESULTS}

Participants' characteristics. A total of 4575 men were diagnosed with prostate cancer after a mean 5.6 years (s.d., 1.0 years) followup. Table 1 shows the characteristics of the study population at baseline. The mean age at recruitment was 56.5 years (s.d., 8.2 years) and the mean BMI was $27.8 \mathrm{~kg} \mathrm{~m}^{-2}$ (s.d., 4.2). Among all participants, $12.4 \%$ reported that they were current cigarette smokers, and $43.2 \%$ reported drinking at least $20 \mathrm{~g}$ of alcohol per day. Physical inactivity was reported by $27.6 \%$ of men. Diabetes was reported by $6.9 \%$ of men. Regarding prostate-specific factors prior to recruitment, $27.6 \%$ of men reported having had at least one PSA test and $7.5 \%$ of men had a family history of prostate cancer.
Table 2 shows the HRs of prostate cancer in relation to sociodemographic factors, anthropometric factors, and lifestyle factors before and after adjusting for multiple factors. There were no marked differences between the minimally and multivariableadjusted models. After multivariable adjustment, men living in North-West England, North-East England, Yorkshire \& the Humber, and in South-West England were significantly less likely to be diagnosed with prostate cancer than men living in London. There was no evidence for an association between living in a deprived area, having higher education, being unemployed, or living with a partner and prostate cancer risk. Compared to men of white ethnicity, Asians had a lower risk $(\mathrm{HR}=0.62$, 95\% CI $0.47-$ 0.83 ) and black men had a higher risk $(2.61,2.10-3.24)$ of prostate cancer.

Height was not associated with prostate cancer risk in the multivariable-adjusted model (Table 2). Obesity (BMI $\geqslant 30-<35$ $v s<25 \mathrm{~kg} \mathrm{~m}^{-2}$ : $\mathrm{HR}=0.88,95 \%$ CI $\left.0.81-0.97\right)$ and morbid obesity $\left(\mathrm{BMI} \geqslant 35\right.$ vs $\left.<25 \mathrm{~kg} \mathrm{~m}^{-2}=0.75,0.64-0.88\right)$, high body fat percentage ( $\mathrm{HR}$ for the highest $v$ s the lowest fifth $=0.81$, 0.73-0.89), high waist circumference (HR for the highest $v s$ the lowest fifth $=0.90,0.82-0.99$ ), and high WHR (HR for the highest $v s$ the lowest fifth $=0.87,0.79-0.96$ ) were all significantly inversely associated with prostate cancer risk. 
Table 2. Hazard ratios $(95 \% \mathrm{CI})$ for prostate cancer in relation to various socio-demographic, anthropometric, and lifestyle factors at baseline

\begin{tabular}{|c|c|c|c|c|c|}
\hline Characteristics & Total $n$ & Cases & $\begin{array}{l}\mathrm{HR}(95 \% \mathrm{Cl}) \text { minimally } \\
\text { adjusted }^{\mathrm{a}}\end{array}$ & $\begin{array}{c}\mathrm{HR}(95 \% \mathrm{Cl}) \\
\text { multivariable-adjusted }^{\mathrm{b}}\end{array}$ & $P$-value \\
\hline \multicolumn{6}{|l|}{ Socio-demographic } \\
\hline Region & & & & & 0.001 \\
\hline Wales & 9075 & 201 & $0.87(0.74-1.02)$ & $0.88(0.75-1.04)$ & \\
\hline North-West England & 35235 & 749 & $0.84(0.76-0.94)$ & $0.86(0.76-0.96)$ & \\
\hline North-Eastern England & 25519 & 480 & $0.77(0.68-0.87)$ & $0.78(0.69-0.89)$ & \\
\hline East Midlands & 14792 & 326 & $0.90(0.78-1.03)$ & $0.89(0.77-1.02)$ & \\
\hline South-East England & 18423 & 471 & $0.98(0.87-1.11)$ & $0.95(0.84-1.08)$ & \\
\hline South-West England & 18181 & 332 & $0.83(0.72-0.95)$ & $0.80(0.70-0.92)$ & \\
\hline Scotland & 15222 & 373 & $0.86(0.75-0.98)$ & $0.87(0.76-1.00)$ & \\
\hline Townsend deprivation score, fifths & & & & & 0.12 \\
\hline One (most affluent) & 43855 & 1020 & 1 ref & 1 ref & \\
\hline Two & 43765 & 1018 & $1.02(0.94-1.11)$ & $1.03(0.94-1.12)$ & \\
\hline No qualifications or CSE/O-Level/GCSE or equivalent & 29593 & 528 & 1 ref & 1 ref & \\
\hline AS/A-Level or equivalent & 11008 & 201 & $1.01(0.86-1.19)$ & $0.99(0.84-1.17)$ & \\
\hline $\begin{array}{l}\text { Higher education or other professional qualification, } \\
\text { or equivalent }\end{array}$ & 137027 & 2797 & $1.07(0.98-1.18)$ & $1.04(0.94-1.14)$ & \\
\hline Ethnicity & & & & & $<0.001$ \\
\hline White & 205839 & 4374 & 1 ref & 1 ref & \\
\hline Mixed background & 1077 & 13 & $0.95(0.55-1.64)$ & $1.01(0.58-1.74)$ & \\
\hline Asian & 5765 & 49 & $0.57(0.43-0.76)$ & $0.62(0.47-0.83)$ & \\
\hline Black & 3279 & 89 & $2.40(1.94-2.97)$ & $2.61(2.10-3.24)$ & \\
\hline Other & 1926 & 23 & $0.94(0.63-1.42)$ & $1.02(0.67-1.54)$ & \\
\hline Unemployment & & & & & 0.719 \\
\hline Paid/self-employment & 134112 & 1904 & 1 ref & 1 ref & \\
\hline Not in paid/self-employment & 85223 & 2671 & $1.00(0.93-1.07)$ & $1.01(0.95-1.08)$ & \\
\hline$\geqslant 180-<185$ & 40076 & 786 & $1.10(1.00-1.21)$ & $1.05(0.95-1.15)$ & \\
\hline$\geqslant 185$ & 21175 & 355 & $1.10(0.97-1.24)$ & $1.04(0.92-1.18)$ & \\
\hline Per $10 \mathrm{~cm}$ increase & 217996 & 4560 & $1.06(1.00-1.11)$ & $1.03(0.98-1.08)$ & 0.307 \\
\hline \multicolumn{6}{|l|}{$\mathrm{BMI}, \mathrm{kg} \mathrm{m}^{-2}$} \\
\hline$<25$ & 53707 & 1142 & 1 ref & 1 ref & \\
\hline$\geqslant 25-<30$ & 107927 & 2396 & $0.98(0.92-1.06)$ & $0.98(0.92-1.06)$ & \\
\hline$\geqslant 30-<35$ & 43500 & 834 & $0.85(0.78-0.93)$ & $0.88(0.81-0.97)$ & \\
\hline$\geqslant 35$ & 12796 & 188 & $0.69(0.59-0.81)$ & $0.75(0.64-0.88)$ & \\
\hline Per $5 \mathrm{~kg} \mathrm{~m}^{-2}$ increase & 217930 & 4560 & $0.89(0.85-0.93)$ & $0.91(0.87-0.95)$ & $<0.001$ \\
\hline \multicolumn{6}{|l|}{ Body fat $^{d}$, fifth cutoff $\%$} \\
\hline$<20.5$ & 43803 & 816 & 1 ref & 1 ref & \\
\hline$\geqslant 20.5-<24$ & 43270 & 938 & $1.02(0.93-1.12)$ & $1.02(0.93-1.12)$ & \\
\hline$\geqslant 24-<26.8$ & 42957 & 949 & $0.95(0.87-1.05)$ & $0.96(0.87-1.06)$ & \\
\hline$\geqslant 26.8-<30.1$ & 41925 & 899 & $0.86(0.78-0.95)$ & $0.88(0.80-0.97)$ & \\
\hline$\geqslant 30.1$ & 42351 & 882 & $0.77(0.70-0.84)$ & $0.81(0.73-0.89)$ & \\
\hline Per $5 \%$ increase & 214257 & 4484 & $0.90(0.88-0.93)$ & $0.92(0.90-0.95)$ & $<0.001$ \\
\hline \multicolumn{6}{|l|}{ Waist circumference, ${ }^{d}$ fifth cutoff $\mathrm{cm}$} \\
\hline$<88$ & 49129 & 939 & 1 ref & 1 ref & \\
\hline$\geqslant 88.1-<93.1$ & 39113 & 826 & $0.99(0.91-1.09)$ & $1.00(0.91-1.10)$ & \\
\hline$\geqslant 93.1-<98.1$ & 49502 & 1113 & $1.00(0.91-1.09)$ & $1.01(0.92-1.10)$ & \\
\hline$\geqslant 98.1-<105.1$ & 37174 & 817 & $0.93(0.85-1.02)$ & $0.96(0.87-1.05)$ & \\
\hline$\geqslant 105.1$ & 43390 & 869 & $0.84(0.76-0.92)$ & $0.90(0.82-0.99)$ & \\
\hline Per $10 \mathrm{~cm}$ increase & 218308 & 4564 & $0.94(0.91-0.97)$ & $0.96(0.93-0.99)$ & 0.016 \\
\hline
\end{tabular}




\section{Table 2. (Continued)}

\begin{tabular}{|c|c|c|c|c|c|}
\hline Characteristics & Total $n$ & Cases & $\begin{array}{c}\mathrm{HR}(95 \% \mathrm{Cl}) \text { minimally } \\
\text { adjusted }^{\mathrm{a}}\end{array}$ & $\begin{array}{c}\text { HR }(95 \% \mathrm{Cl}) \\
\text { multivariable-adjusted }^{\mathrm{b}}\end{array}$ & $P$-value ${ }^{c}$ \\
\hline $\begin{array}{l}\text { Waist to hip ratio, }{ }^{\mathbf{d}} \text { fifth cutoff } \\
\quad<0.882 \\
\geqslant 0.882-<0.918 \\
\geqslant 0.918-<0.949 \\
\geqslant 0.949-<0.989 \\
\geqslant 0.989 \\
\text { Per } 0.05 \text { increase }\end{array}$ & $\begin{array}{r}43848 \\
43787 \\
43872 \\
43110 \\
43639 \\
218256\end{array}$ & $\begin{array}{r}835 \\
835 \\
982 \\
964 \\
948 \\
4564\end{array}$ & $\begin{array}{c}1 \text { ref } \\
0.87(0.79-0.96) \\
0.94(0.86-1.03) \\
0.87(0.79-0.96) \\
0.80(0.73-0.88) \\
0.94(0.92-0.97)\end{array}$ & $\begin{array}{c}1 \text { ref } \\
0.88(0.80-0.97) \\
0.96(0.88-1.06) \\
0.91(0.83-1.00) \\
0.87(0.79-0.96) \\
0.97(0.94-1.00)\end{array}$ & 0.021 \\
\hline $\begin{array}{l}\text { Alcohol intake, g per day } \\
\text { None drinkers } \\
\quad<1 \\
\geqslant 1-<10 \\
\geqslant 10-<20 \\
\geqslant 20 \\
\text { Per } 10 \text { g per day increase }\end{array}$ & $\begin{array}{r}13874 \\
14577 \\
48187 \\
46565 \\
94750 \\
204723\end{array}$ & $\begin{array}{r}225 \\
299 \\
1030 \\
1071 \\
1930 \\
4340\end{array}$ & $\begin{array}{c}0.83(0.70-0.99) \\
1 \text { ref } \\
1.08(0.95-1.23) \\
1.11(0.98-1.26) \\
1.02(0.90-1.15) \\
0.98(0.96-1.00)\end{array}$ & $\begin{array}{l}0.85(0.72-1.01) \\
1 \text { ref } \\
1.04(0.91-1.18) \\
1.06(0.93-1.21) \\
1.01(0.89-1.14) \\
0.99(0.97-1.01)\end{array}$ & 0.405 \\
\hline $\begin{array}{l}\text { Physical activity, METs per week } \\
\text { Low, } 0-<10 \\
\text { Moderate, } \geqslant 10-<50 \\
\text { High, } \geqslant 50 \\
\text { Per } 20 \text { METs per week increase }\end{array}$ & $\begin{array}{r}60589 \\
103570 \\
47126 \\
211070\end{array}$ & $\begin{array}{r}1196 \\
2244 \\
975 \\
4413 \\
\end{array}$ & $\begin{array}{c}1 \text { ref } \\
1.10(1.02-1.18) \\
1.05(0.96-1.14) \\
1.00(0.98-1.03)\end{array}$ & $\begin{array}{c}1 \text { ref } \\
1.06(0.99-1.14) \\
1.01(0.93-1.10) \\
1.00(0.98-1.02)\end{array}$ & 0.771 \\
\hline 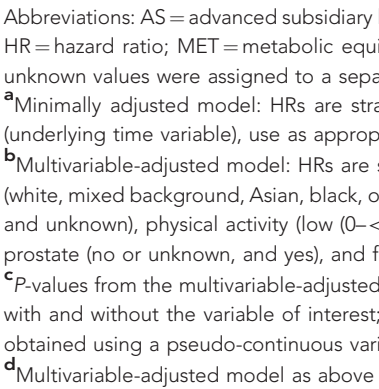 & $\begin{array}{l}l=\text { confide } \\
\text { Missing an } \\
\text { le was inclu } \\
r \text { registry re } \\
\text { recruitmen } \\
\text { wife or par } \\
\text { ( } \geqslant 10-<5 \\
r \text { (no, yes, } \\
\text { ows: } P \text {-value } \\
\text { lables were } \\
\text { e in each c } \\
\text { ll. }\end{array}$ & $\begin{array}{l}\text { e interval; } \\
\text { or unknow } \\
\text { ed as a cove } \\
\text { ions) and a } \\
\text { and adjuste } \\
\text { er (no and y } \\
\text { METs per w } \\
\text { nnown), use } \\
\text { for likelihoc } \\
\text { otained cor } \\
\text { egory for cc }\end{array}$ & $\begin{array}{l}=\text { Certificate of Secondary Educat } \\
\text { lues of the exposure of interest } \\
\text { e. } \\
\text { at recruitment }(<45,45-49,50-5 \\
\text { or age (underlying time variable), } \\
\text { BMI ( }<25, \geqslant 25-<30, \geqslant 30-<35 \text {, } \\
\text {, high }(\geqslant 50 \mathrm{MET} \text { per week), an } \\
\text { appropriate. } \\
\text { atio test for variables with more th } \\
\text { ring the reference category to th } \\
\text { uous variables. }\end{array}$ & $\begin{array}{l}\text { GCSE }=\text { General Certificate of Seco } \\
\text { e not included in the analyses, bu } \\
5-59,60-64 \text {, and } \geqslant 65 \text { years) and } \\
\text { unsend deprivation score (fifths, un } \\
5 \mathrm{~kg} \mathrm{~m}^{-2} \text {, unknown), smoking (neve } \\
\text { known), diabetes (no, yes, and un } \\
\text { two categories were obtained com } \\
\text { ther category in the model; } P \text {-value }\end{array}$ & $\begin{array}{l}\text { y Education; } \\
\text { ssing and/or } \\
\text { sted for age } \\
\text { vn), ethnicity } \\
\text { mer, current, } \\
\text { un), enlarged } \\
g \text { the model } \\
\text { r trend were }\end{array}$ \\
\hline
\end{tabular}

Among the lifestyle characteristics analysed in this study, compared with never smokers, current cigarette smokers $(0.85$, $0.77-0.95)$ and former cigarette smokers $(0.93,0.88-0.99)$ had a significantly lower risk of prostate cancer, while no association with risk was observed for alcohol intake or physical activity.

Table 3 shows the HRs of prostate cancer in relation to health status and prostate-specific factors prior to recruitment, sexual history, early life factors, and hair colour and balding pattern. Men with a self-reported diagnosis of diabetes had a lower risk of incident prostate cancer $(\mathrm{HR}=0.70,0.62-0.80)$. In contrast, men who had had a PSA test prior to recruitment $(1.31,1.23-1.40)$, had any first-degree family history of prostate cancer (1.94, 1.77-2.13), and who reported that they had been diagnosed with an enlarged prostate $(1.54,1.38-1.71)$ had an elevated risk of prostate cancer. Moreover, compared to men with no family history of prostate cancer, men with both their father and brother diagnosed with prostate cancer had an even higher risk of prostate cancer (3.35, $2.33-4.81)$.

For sexual history factors, men who had no children had a reduced prostate cancer risk (never vs ever, $\mathrm{HR}=0.89,95 \% \mathrm{CI}$ 0.81-0.97), as did men who reported they had never had sex (never vs ever, $0.53,0.33-0.84)$. Other sexual history characteristics were not related to prostate cancer risk (Table 3 ).

Early life factors (relative age of first facial hair, relative age voice broke, and comparative body size and height at age 10) and hair colour and pattern were not associated with prostate cancer risk (Table 3).

For most factors, there was no significant heterogeneity in the association of prostate cancer risk according to time between recruitment and diagnosis ( $<2$ and $\geqslant 2$ years) (Supplementary Table 1). However, there was evidence of heterogeneity by time to diagnosis for the association of prostate cancer risk with ethnicity ( $P_{\text {heterogeneity }}=0.001$; for black $v s$ white, $\mathrm{HR}=3.94,2.85-5.44$ for men diagnosed within 2 years and $\mathrm{HR}=2.01,1.49-2.70$ for those diagnosed after 2 years), unemployment $\left(P_{\text {heterogeneity }}<0.001\right.$; $\mathrm{HR}=1.34,1.18-1.51$ in the first 2 years and $\mathrm{HR}=0.89,0.82$ 0.97 after 2 years $)$, hypertension $\left(P_{\text {heterogeneity }}=0.018\right.$; for yes $v s$ no, $\mathrm{HR}=1.12,1.00-1.24$ in the first 2 years and $\mathrm{HR}=0.95,0.89-1.02$ after 2 years), having had a PSA test prior to recruitment $\left(P_{\text {heterogeneity }}=0.003 ; \mathrm{HR}=1.51,1.35-1.70\right.$ in the first 2 years and $1.23,1.14-1.33$ after 2 years), and having had enlarged prostate ( $P_{\text {heterogeneity }}<0.001 ; \mathrm{HR}=2.16,1.82-2.56$ in the first 2 years and $1.27,1.10-1.46$ after 2 years; Supplementary Table 1).

\section{DISCUSSION}

Here we report results on associations with established and putative risk factors for prostate cancer risk in a large prospective study of British men. We found that black ethnicity and having previously had a PSA test, an enlarged prostate, or a family history of prostate cancer were positively associated with prostate cancer risk. The risk of being diagnosed with prostate cancer was lower in those who were of Asian ethnic origin, and in men who had obesity, smoked cigarettes, had diabetes, and had never had sex. Time to diagnosis was not a strong modifier of these associations, although men who had had a PSA test prior to recruitment were 
Table 3. Hazard ratios $(95 \% \mathrm{Cl})$ for prostate cancer in relation to various health status, prostate specific factors prior to recruitment, sexual history, and early life factors at baseline

\begin{tabular}{|c|c|c|c|c|c|}
\hline Characteristics & Total $n$ & Cases & $\begin{array}{c}\mathrm{HR}(95 \% \mathrm{Cl}) \text { minimally } \\
\text { adjusted }^{\mathrm{a}}\end{array}$ & $\begin{array}{c}\mathrm{HR}(95 \% \mathrm{Cl}) \\
\text { multivariable-adjusted }^{\mathrm{b}}\end{array}$ & $P$-value ${ }^{c}$ \\
\hline \multicolumn{6}{|l|}{ Health status } \\
\hline $\begin{array}{l}\text { Vasectomy } \\
\text { No or unknown } \\
\text { Yes }\end{array}$ & $\begin{array}{r}207969 \\
11366\end{array}$ & $\begin{array}{r}4343 \\
232\end{array}$ & $\begin{array}{c}1 \mathrm{ref} \\
1.05(0.92-1.20)\end{array}$ & $\begin{array}{c}1 \mathrm{ref} \\
1.03(0.90-1.18)\end{array}$ & 0.662 \\
\hline $\begin{array}{l}\text { Hypertension } \\
\text { No or unknown } \\
\text { Yes }\end{array}$ & $\begin{array}{l}104608 \\
114193\end{array}$ & $\begin{array}{l}1866 \\
2700\end{array}$ & $\begin{array}{c}1 \text { ref } \\
0.99(0.93-1.05)\end{array}$ & $\begin{array}{c}1 \mathrm{ref} \\
1.00(0.94-1.06)\end{array}$ & 0.965 \\
\hline \multicolumn{6}{|l|}{ Prostate-specific factors prior to recruitment } \\
\hline $\begin{array}{l}\text { PSA test } \\
\text { No } \\
\text { Yes }\end{array}$ & $\begin{array}{r}154265 \\
58497\end{array}$ & $\begin{array}{l}2283 \\
2009\end{array}$ & $\begin{array}{c}1 \text { ref } \\
1.43(1.35-1.52)\end{array}$ & $\begin{array}{c}1 \mathrm{ref} \\
1.31(1.23-1.40)\end{array}$ & $<0.001$ \\
\hline $\begin{array}{l}\text { Any first-degree family history of prostate cancer } \\
\text { No } \\
\text { Yes }\end{array}$ & $\begin{array}{r}103961 \\
16449 \\
\end{array}$ & $\begin{array}{r}1745 \\
599 \\
\end{array}$ & $\begin{array}{c}1 \mathrm{ref} \\
1.96(1.78-2.15)\end{array}$ & $\begin{array}{c}1 \mathrm{ref} \\
1.94(1.77-2.13)\end{array}$ & $<0.001$ \\
\hline $\begin{array}{l}\text { Family history of prostate cancer } \\
\text { No } \\
\text { Father or brother } \\
\text { Father and brother }\end{array}$ & $\begin{array}{r}103961 \\
16098 \\
351\end{array}$ & $\begin{array}{r}1745 \\
569 \\
30\end{array}$ & $\begin{array}{c}1 \text { ref } \\
1.91(1.74-2.10) \\
3.51(2.45-5.04)\end{array}$ & $\begin{array}{c}1 \text { ref } \\
1.90(1.72-2.09) \\
3.35(2.33-4.81)\end{array}$ & $<0.001$ \\
\hline \multicolumn{6}{|l|}{ Sexual history } \\
\hline $\begin{array}{l}\text { Ever had children } \\
\text { Yes } \\
\text { Never }\end{array}$ & $\begin{array}{r}171957 \\
45375\end{array}$ & $\begin{array}{r}3879 \\
669\end{array}$ & $\begin{array}{c}1 \text { ref } \\
0.87(0.80-0.94)\end{array}$ & $\begin{array}{c}1 \text { ref } \\
0.89(0.81-0.97)\end{array}$ & 0.007 \\
\hline $\begin{array}{l}\text { Number of children } \\
1 \\
2 \\
\geqslant 3\end{array}$ & $\begin{array}{l}27488 \\
90098 \\
54371\end{array}$ & $\begin{array}{r}517 \\
2131 \\
1231\end{array}$ & $\begin{array}{c}0.94(0.85-1.03) \\
1 \text { ref } \\
0.92(0.86-0.99)\end{array}$ & $\begin{array}{c}0.95(0.86-1.04) \\
1 \text { ref } \\
0.93(0.86-1.00)\end{array}$ & 0.001 \\
\hline $\begin{array}{l}\text { Lifetime number of heterosexual partners } \\
\qquad \begin{array}{l}1 \\
\geqslant 2-<6 \\
\geqslant 6\end{array}\end{array}$ & $\begin{array}{l}41598 \\
65701 \\
68971\end{array}$ & $\begin{array}{l}1126 \\
1392 \\
1161\end{array}$ & $\begin{array}{c}1 \text { ref } \\
0.96(0.89-1.04) \\
0.99(0.91-1.07)\end{array}$ & $\begin{array}{c}1 \text { ref } \\
0.99(0.91-1.07) \\
1.03(0.95-1.13)\end{array}$ & 0.013 \\
\hline $\begin{array}{l}\text { Same-sex intercourse } \\
\text { No } \\
\text { Yes }\end{array}$ & $\begin{array}{r}189406 \\
8576\end{array}$ & $\begin{array}{r}3989 \\
146\end{array}$ & $\begin{array}{c}1 \mathrm{ref} \\
1.07(0.90-1.26)\end{array}$ & $\begin{array}{c}1 \mathrm{ref} \\
1.12(0.95-1.33)\end{array}$ & 0.171 \\
\hline $\begin{array}{l}\text { Lifetime number of same-sex partners } \\
\qquad \begin{array}{l}1 \\
\geqslant 2-<6 \\
\geqslant 6\end{array}\end{array}$ & $\begin{array}{r}977 \\
1340 \\
1716\end{array}$ & $\begin{array}{l}18 \\
25 \\
22\end{array}$ & $\begin{array}{c}1 \text { ref } \\
1.05(0.57-1.92) \\
0.94(0.50-1.75)\end{array}$ & $\begin{array}{c}1 \text { ref } \\
1.05(0.57-1.92) \\
0.93(0.50-1.74)\end{array}$ & 0.964 \\
\hline \multicolumn{6}{|l|}{ Early life } \\
\hline $\begin{array}{l}\text { Relative age of first facial hair } \\
\text { Younger than average } \\
\text { About average } \\
\text { Older than average }\end{array}$ & $\begin{array}{r}14262 \\
167220 \\
27009 \\
\end{array}$ & $\begin{array}{r}243 \\
3656 \\
459 \\
\end{array}$ & $\begin{array}{c}1.01(0.89-1.15) \\
1 \text { ref } \\
0.97(0.88-1.06)\end{array}$ & $\begin{array}{c}1.03(0.90-1.17) \\
1 \text { ref } \\
0.94(0.86-1.04)\end{array}$ & 0.454 \\
\hline $\begin{array}{l}\text { Relative age voice broke } \\
\text { Younger than average } \\
\text { About average } \\
\text { Older than average }\end{array}$ & $\begin{array}{r}8769 \\
178309 \\
11861\end{array}$ & $\begin{array}{r}143 \\
3813 \\
194\end{array}$ & $\begin{array}{c}0.99(0.84-1.17) \\
1 \text { ref } \\
0.95(0.83-1.10)\end{array}$ & $\begin{array}{c}1.00(0.85-1.18) \\
1 \text { ref } \\
0.92(0.80-1.07)\end{array}$ & 0.548 \\
\hline $\begin{array}{l}\text { Comparative body size at age } 10 \\
\text { Thinner } \\
\text { About average } \\
\text { Plumper }\end{array}$ & $\begin{array}{r}75069 \\
109231 \\
29305\end{array}$ & $\begin{array}{r}1516 \\
2414 \\
536\end{array}$ & $\begin{array}{c}0.94(0.88-1.00) \\
1 \text { ref } \\
0.92(0.84-1.01)\end{array}$ & $\begin{array}{c}0.94(0.88-1.00) \\
1 \text { ref } \\
0.96(0.88-1.06)\end{array}$ & 0.156 \\
\hline
\end{tabular}




\section{Table 3. (Continued)}

\begin{tabular}{|c|c|c|c|c|c|}
\hline Characteristics & Total $n$ & Cases & $\begin{array}{c}\mathrm{HR}(95 \% \mathrm{Cl}) \text { minimally } \\
\text { adjusted }^{\mathrm{a}}\end{array}$ & $\begin{array}{c}\text { HR }(95 \% \mathrm{Cl}) \\
\text { multivariable-adjusted }^{\mathrm{b}}\end{array}$ & $P$-value ${ }^{c}$ \\
\hline $\begin{array}{l}\text { Comparative height size at age } 10 \\
\text { Shorter } \\
\text { About average } \\
\text { Taller }\end{array}$ & $\begin{array}{r}42033 \\
119071 \\
53953\end{array}$ & $\begin{array}{r}865 \\
2510 \\
1115\end{array}$ & $\begin{array}{c}1.00(0.93-1.08) \\
1 \text { ref } \\
1.01(0.94-1.09)\end{array}$ & $\begin{array}{c}1.00(0.92-1.08) \\
1 \text { ref } \\
1.00(0.94-1.08)\end{array}$ & 0.986 \\
\hline \multicolumn{6}{|l|}{ Hair colour and pattern } \\
\hline $\begin{array}{l}\text { Hair colour (natural, before greying in whites) } \\
\text { Light brown } \\
\text { Red } \\
\text { Blonde } \\
\text { Dark brown } \\
\text { Black } \\
\text { Other }\end{array}$ & $\begin{array}{r}79825 \\
7726 \\
19935 \\
77304 \\
16718 \\
17152\end{array}$ & $\begin{array}{r}1790 \\
144 \\
418 \\
1523 \\
406 \\
287\end{array}$ & $\begin{array}{c}1 \text { ref } \\
0.88(0.75-1.05) \\
0.95(0.86-1.06) \\
0.98(0.91-1.05) \\
1.02(0.92-1.14) \\
1.01(0.89-1.14)\end{array}$ & $\begin{array}{c}1 \text { ref } \\
0.88(0.74-1.05) \\
0.96(0.86-1.06) \\
0.97(0.91-1.04) \\
1.03(0.92-1.14) \\
0.99(0.79-1.23)\end{array}$ & 0.597 \\
\hline $\begin{array}{l}\text { Hair/balding pattern } \\
\text { Pattern } 1 \\
\text { Pattern } 2 \\
\text { Pattern } 3 \\
\text { Pattern } 4\end{array}$ & $\begin{array}{l}69647 \\
49303 \\
57208 \\
39051\end{array}$ & $\begin{array}{r}1317 \\
903 \\
1388 \\
882 \\
\end{array}$ & $\begin{array}{c}1 \text { ref } \\
0.99(0.91-1.08) \\
1.00(0.93-1.08) \\
0.95(0.87-1.03)\end{array}$ & $\begin{array}{c}1 \text { ref } \\
0.99(0.91-1.07) \\
0.99(0.92-1.07) \\
0.95(0.87-1.03)\end{array}$ & 0.595 \\
\hline \multicolumn{6}{|c|}{ 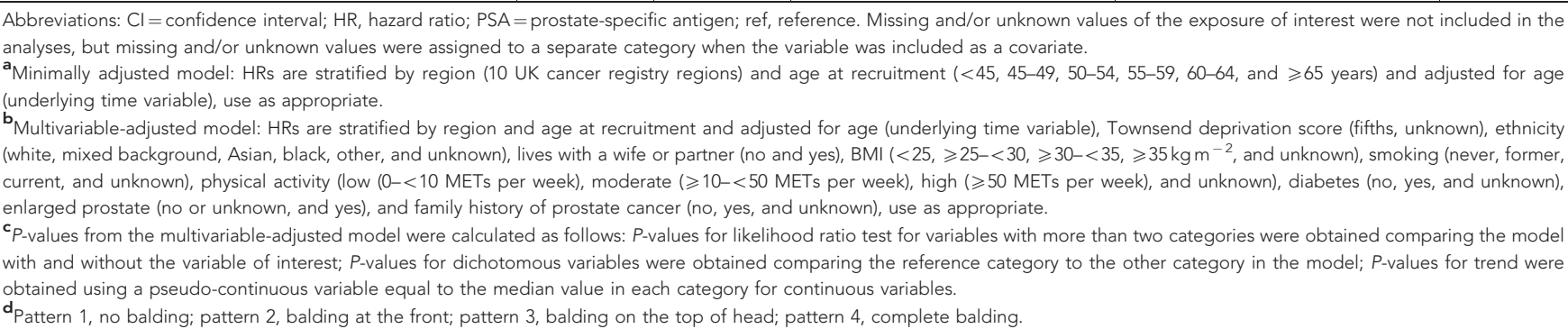 } \\
\hline
\end{tabular}

more likely to be diagnosed with prostate cancer in the first 2 years of follow-up.

Ethnicity, socio-demographic, anthropometric and lifestyle factors, and risk of prostate cancer. Our findings for ethnicity accord with findings from the retrospective PROCESS cohort study, which has previously shown that compared to white men, black men in southern England have a greater risk of being diagnosed with prostate cancer (Ben-Shlomo et al, 2008), while Asian men have a lower risk (Metcalfe et al, 2008). A crosssectional study within the Hospital Episodes Statistics database for England also showed a higher risk of prostate cancer in men with black ethnicity (Maruthappu et al, 2015).

Geographic differences in prostate cancer incidence rates have been observed in the United States (Cook et al, 2015), indicating that risk factors for prostate cancer occurrence and for diagnosis may vary geographically. In the current British study, there were differences in risk between certain geographical regions. It is possible that some of the regional differences might be due to differences in detection rates of asymptomatic prostate cancer (Littlejohns et al, 2016). Socioeconomic status, education level, employment status, and marital status (living with a wife or partner) were not associated with prostate cancer risk.

While we did not observe a significant association of prostate cancer risk with height, as seen in previous prospective studies (Pischon et al, 2008; WCRF/AICR, 2014), our results did show that men with higher BMI and fat mass percentage had a lower risk of prostate cancer. While two previous prospective studies have used fat mass measurement from bioimpedance measurements (477 (MacInnis et al, 2003) and 817 incident cases (Wallstrom et al, 2009), respectively), this is to our knowledge the first large prospective study using bioimpedance to estimate body composition. Previous prospective investigations have also found a link between excess adiposity, typically as estimated by BMI or waist circumference, and a lower risk of overall prostate cancer risk
(Perez-Cornago et al, 2017; WCRF/AICR, 2014). It is possible that this association might be due to detection bias as in this cohort men with obesity are less likely to have had a PSA test (Littlejohns et al, 2016). Moreover, previous studies have reported slightly lower PSA concentrations in men with high BMI (Bonn et al, 2016). A positive association between adiposity and risk for aggressive prostate cancer has also been observed in previous studies (WCRF/AICR, 2014), but data on stage and grade are not yet available in the UK Biobank cohort.

Findings from the present study of a nearly $15 \%$ reduced risk of prostate cancer in cigarette smokers compared to never smokers are consistent with results from a 2010 meta-analysis of 24 observational studies (Huncharek et al, 2010). However, men who were current smokers were markedly less likely to have had a PSA test than never smokers in UK Biobank (Littlejohns et al, 2016), and this association might therefore be due to detection bias.

In agreement with findings from a recent meta-analysis (WCRF/AICR, 2014), alcohol consumption was not related to prostate cancer risk in the current study. Total physical activity was also not associated with prostate cancer risk in the current study, whereas findings from a recent meta-analysis of 46890 incident cases, which showed that greater leisure-time physical activity was associated with a higher risk of prostate cancer (Moore et al, 2016), although the impact on those results of detection bias was not clear (i.e., the extent to which PSA testing is associated with healthconscious behaviour).

Health status, prostate-specific factors prior to recruitment, sexual history, early life factors, and hair colour and pattern. In agreement with previous studies (Byrne et al, 2017; Nayan et al, 2016), we found no association between vasectomy status and prostate cancer risk. In the current study, hypertension was not linked to prostate cancer risk, although there was some evidence that it was associated with an increased risk in the first 2 years of follow-up. A recent meta-analysis has suggested that hypertension may be related to prostate cancer incidence, but high heterogeneity 
among studies was noted (Liang et al, 2016), and more prospective data are needed. Our finding that diabetes was associated with a reduced risk of prostate cancer has been consistently reported in other cohort studies (Rodriguez et al, 2005; Tsilidis et al, 2015). It has been suggested that the inverse association between diabetes and prostate cancer risk might be due to lower circulating concentrations of IGF-I (Teppala \& Shankar, 2010) and/or testosterone (Grossmann, 2011) or to potential anti-carcinogenic properties of diabetes medication (Wright and Stanford, 2009). Information was not available on diabetes type for the current analyses, but the majority of cases in this age group will be of type II diabetes (Kirkman et al, 2012).

Increases in the proportion of men undergoing PSA testing in the UK have led to a large increase in prostate cancer diagnoses over recent decades (Lilja et al, 2008), and as expected, history of having had a PSA test was positively associated with prostate cancer risk in our study. As expected, previous PSA testing was also more strongly associated with risk in the first 2 years of follow-up, owing to it being a first-line test in the diagnostic pathway for men with prostatic symptoms. Similarly, men with an enlarged prostate were also more likely to be diagnosed with prostate cancer, particularly within the first 2 years, suggesting increased likelihood of cancer detection following urological investigations.

This study found that having a first-degree relative with prostate cancer doubled the risk of prostate cancer, which is wellestablished, although to date only approximately one-third of this risk is explained by known genetic variants (Benafif \& Eeles, 2016). The risk was even higher in men where both the father and the brother had prostate cancer, although the number of cases in this category was small $(n=30)$. The remainder of the excess familial risk may be due to a combination of currently unidentified genetic variation, shared environmental factors, and differential detection in family members (e.g., health-seeking behaviours).

There are few prospective data on sexual history and prostate cancer risk (Rosenblatt et al, 2001). Our results show that men who reported never having had sex have a lower prostate cancer risk than men who had ever had sex. Moreover, men who had not had children had a lower risk compared to those who had had children. Men who have not had sex might have erectile dysfunction or low sexual interest, which are both conditions that have been linked to reduced circulating androgen levels (Isidori et al, 2005). Observational epidemiological studies to date have not shown an association between circulating testosterone and prostate cancer risk (Roddam et al, 2008), but more data are required to examine risk in men with very low circulating testosterone levels.

To date it is unclear whether early-life exposures are involved in prostate cancer aetiology (Sutcliffe \& Colditz, 2013; Moller et al, 2015; Sarre et al, 2016), although it has been speculated that childhood body size or timing of puberty may be related to changes in prostate tissue in early adulthood (Sutcliffe \& Colditz, 2013; Sarre et al, 2016). Mendelian randomisation studies have shown an association between genetically determined age at puberty and higher risk of aggressive prostate cancer risk (Bonilla et al, 2016). In the current study, however, we found no evidence for an association between a number of early life factors (relative age of first facial hair, relative age voice broke, and comparative height and body size at age 10) and the future risk of prostate cancer.

It has been hypothesised that pigmentation-related traits may influence prostate cancer risk, possibly through altered vitamin D synthesis, owing to a finding in the Alpha-Tocopherol, BetaCarotene Cancer Prevention Study that men with naturally red hair (which is determined by polymorphisms in the melanocortin1-receptor $(M C 1 R)$ gene) had a lower risk of prostate cancer compared to men with light brown hair (Weinstein et al, 2013); however, no significant association between naturally red hair and prostate cancer risk was observed in the current study. Because of the influence of the active androgen dihydrotestosterone on both the growth of prostate cells and on androgenic alopecia, malepattern balding has also been suggested as possible risk factor for prostate cancer, although the findings are inconclusive (Muller et al, 2013; Zhou et al, 2015a, b; Sarre et al, 2016) and the current study found no association between balding pattern and prostate cancer risk.

Study strengths and limitations. This is, to our knowledge, the largest single prospective study of risk factors for prostate cancer in British men. The UK Biobank has collected detailed information on numerous possible prostate cancer risk factors, including risk factors that few previous studies have examined in detail, such as body fat mass, sexual history, early life factors, and hair colour and pattern. In particular, fat mass estimated using bioimpedance is a better marker of overall adiposity than BMI or waist circumference, which does not differentiate between muscle and fat mass. Moreover, many risk factors, such as adiposity measurements or blood pressure, were assessed by trained research clinic staff instead of being self-reported.

Despite the breadth of the exposure information collected at recruitment, we cannot exclude the possibility of residual confounding by unknown or unmeasured factors. In addition, because of the number of tests performed, some of the associations observed might be due to chance. For some of the rare exposures (e.g., red hair colour or never having had sexual intercourse), there are small numbers of exposed cases for robust analysis. This cohort includes participants from multiple regions, including deprived areas, but it is not a representative sample of the whole UK population (Fry et al, 2017). However, it does include participants with a wide range of exposures for a comprehensive set of characteristics allowing internally valid and informative comparisons of risk by factors of interest. Although the number of missing values in our cohort is low $(<1 \%)$, there are some variables, such as family history of prostate cancer, that have a higher proportion of missing values. These values may not be missing at random, for example, participants with family history of prostate cancer may have replied to this question, while participants with no family history may have left this question blank. Finally, risk factors for prostate cancer may differ by tumour characteristics, but data on tumour stage and Gleason grade were not available for the current analysis. We will perform analyses by subgroups of disease aggressiveness when these data become available.

We have reported a range of established and novel risk factors that are associated with subsequent prostate cancer risk in this large UK prospective study. In particular, black ethnicity, having had a PSA test, an enlarged prostate, and a family history of prostate cancer were positively associated with prostate cancer risk, while Asian ethnicity, obesity, smoking status, diabetes, and never having had children or sexual intercourse were related to a lower prostate cancer risk. Future research in UK Biobank will include analyses by disease aggressiveness to explore whether these associations are due to differences in the likelihood of being diagnosed and/or differences in the risk of developing clinically important prostate cancer.

\section{ACKNOWLEDGEMENTS}

These analyses were supported by Cancer Research UK (C8221/ A19170). KEB is supported by a Girdlers' New Zealand Health Research Council Fellowship. RMM was supported by CRUK grant number C18281/A19169 (the Integrative Cancer Epidemiology Programme). This work has been conducted using the UK Biobank 
Resource under Application Number 3282 and we express our gratitude to the participants and those involved in building the resource.

\section{CONFLICT OF INTEREST}

The authors declare no conflict of interest.

\section{REFERENCES}

Ben-Shlomo Y, Evans S, Ibrahim F, Patel B, Anson K, Chinegwundoh F, Corbishley C, Dorling D, Thomas B, Gillatt D, Kirby R, Muir G, Nargund V, Popert R, Metcalfe C, Persad R, group Ps (2008) The risk of prostate cancer amongst black men in the United Kingdom: the PROCESS cohort study. Eur Urol 53(1): 99-105.

Benafif S, Eeles R (2016) Genetic predisposition to prostate cancer. $\mathrm{Br}$ Med Bull 120(1): 75-89.

Bonilla C, Lewis SJ, Martin RM, Donovan JL, Hamdy FC, Neal DE, Eeles R, Easton D, Kote-Jarai Z, Al Olama AA, Benlloch S, Muir K, Giles GG, Wiklund F, Gronberg H, Haiman CA, Schleutker J, Nordestgaard BG, Travis RC, Pashayan N, Khaw KT, Stanford JL, Blot WJ, Thibodeau S, Maier C, Kibel AS, Cybulski C, Cannon-Albright L, Brenner H, Park J, Kaneva R, Batra J, Teixeira MR, Pandha H, Lathrop M, Davey Smith G, consortium P (2016) Pubertal development and prostate cancer risk: Mendelian randomization study in a population-based cohort. BMC Med 14: 66.

Bonn SE, Sjolander A, Tillander A, Wiklund F, Gronberg H, Balter K (2016) Body mass index in relation to serum prostate-specific antigen levels and prostate cancer risk. Int J Cancer 139(1): 50-57.

Byrne KS et al. (2017) Vasectomy and prostate cancer risk in the European Prospective Investigation into Cancer and Nutrition (EPIC). J Clin Oncol 35: 1297-1303.

Cook MB, Rosenberg PS, McCarty FA, Wu M, King J, Eheman C, Anderson WF (2015) Racial disparities in prostate cancer incidence rates by census division in the United States, 1999-2008. Prostate 75(7): 758-763.

Cuzick J, Thorat MA, Andriole G, Brawley OW, Brown PH, Culig Z, Eeles RA, Ford LG, Hamdy FC, Holmberg L, Ilic D, Key TJ, La Vecchia C, Lilja H, Marberger M, Meyskens FL, Minasian LM, Parker C, Parnes HL, Perner S, Rittenhouse H, Schalken J, Schmid HP, Schmitz-Drager BJ, Schroder FH, Stenzl A, Tombal B, Wilt TJ, Wolk A (2014) Prevention and early detection of prostate cancer. Lancet Oncol 15(11): e484-e492.

Ferlay J, Soerjomataram I, Dikshit R, Eser S, Mathers C, Rebelo M, Parkin DM, Forman D, Bray F (2015) Cancer incidence and mortality worldwide: sources, methods and major patterns in GLOBOCAN 2012. Int J Cancer 136(5): E359-E386.

Fry A, Littlejohns TJ, Sudlow C, Doherty N, Adamska L, Sprosen T, Collins R, Allen NE (2017) Comparison of sociodemographic and health-related characteristics of uk biobank participants with the general population. Am J Epidemiol. e-pub ahead of print 21 Jun 2017; doi:10.1093/aje/ kwx246.

Grossmann M (2011) Low testosterone in men with type 2 diabetes: significance and treatment. J Clin Endocrinol Metab 96(8): 2341-2353.

Huncharek M, Haddock KS, Reid R, Kupelnick B (2010) Smoking as a risk factor for prostate cancer: a meta-analysis of 24 prospective cohort studies. Am J Public Health 100(4): 693-701.

Isidori AM, Giannetta E, Gianfrilli D, Greco EA, Bonifacio V, Aversa A, Isidori A, Fabbri A, Lenzi A (2005) Effects of testosterone on sexual function in men: results of a meta-analysis. Clin Endocrinol (Oxf) 63(4): 381-394.

Kirkman MS, Briscoe VJ, Clark N, Florez H, Haas LB, Halter JB, Huang ES, Korytkowski MT, Munshi MN, Odegard PS, Pratley RE, Swift CS (2012) Diabetes in older adults. Diabetes Care 35(12): 2650-2664.

Liang Z, Xie B, Li J, Wang X, Wang S, Meng S, Ji A, Zhu Y, Xu X, Zheng X, Xie L (2016) Hypertension and risk of prostate cancer: a systematic review and meta-analysis. Sci Rep 6: 31358.

Lilja H, Ulmert D, Vickers AJ (2008) Prostate-specific antigen and prostate cancer: prediction, detection and monitoring. Nat Rev Cancer 8(4): $268-278$.
Littlejohns TJ, Travis RC, Key TJ, Allen NE (2016) Lifestyle factors and prostate-specific antigen (PSA) testing in UK Biobank: Implications for epidemiological research. Cancer Epidemiol 45: 40-46.

MacInnis RJ, English DR, Gertig DM, Hopper JL, Giles GG (2003) Body size and composition and prostate cancer risk. Cancer Epidemiol Biomarkers Prev 12(12): 1417-1421.

Maruthappu M, Barnes I, Sayeed S, Ali R (2015) Incidence of prostate and urological cancers in England by ethnic group, 2001-2007: a descriptive study. BMC Cancer 15: 753.

Metcalfe C, Patel B, Evans S, Ibrahim F, Anson K, Chinegwundoh F, Corbishley C, Dorling D, Thomas B, Gillatt D, Kirby R, Muir G, Nargund V, Popert R, Persad R, Ben-Shlomo Y, Group PS (2008) The risk of prostate cancer amongst South Asian men in southern England: the PROCESS cohort study. BJU Int 102(10): 1407-1412.

Moller E, Wilson KM, Batista JL, Mucci LA, Balter K, Giovannucci E (2015) Body size across the life course and prostate cancer in the Health Professionals Follow-up Study. Int J Cancer 138(4): 853-865.

Moore SC, Lee IM, Weiderpass E, Campbell PT, Sampson JN, Kitahara CM, Keadle SK, Arem H, Berrington de Gonzalez A, Hartge P, Adami HO, Blair CK, Borch KB, Boyd E, Check DP, Fournier A, Freedman ND, Gunter M, Johannson M, Khaw KT, Linet MS, Orsini N, Park Y, Riboli E, Robien K, Schairer C, Sesso H, Spriggs M, Van Dusen R, Wolk A, Matthews CE, Patel AV (2016) Association of leisure-time physical activity with risk of 26 types of cancer in 1.44 million adults. JAMA Intern Med 176(6): 816-825.

Muller DC, Giles GG, Sinclair R, Hopper JL, English DR, Severi G (2013) Agedependent associations between androgenetic alopecia and prostate cancer risk. Cancer Epidemiol Biomarkers Prev 22(2): 209-215.

Nayan M, Hamilton RJ, Macdonald EM, Li Q, Mamdani MM, Earle CC, Kulkarni GS, Jarvi KA, Juurlink DN, Canadian Drug SEffectiveness Research N (2016) Vasectomy and risk of prostate cancer: population based matched cohort study. BMJ 355: i5546.

ONS (2017) Office for National Statistics: Cancer registration statistics, England: first release, 2015. Available at: https://www.ons.gov.uk/people populationandcommunity/healthandsocialcare/conditionsanddiseases/ bulletins/cancerregistrationstatisticsengland/firstrelease2015 (last accessed 20 April 2017).

Perez-Cornago A, Appleby PN, Pischon T, Tsilidis KK, Tjonneland A, Olsen A, Overvad K, Kaaks R, Kuhn T, Boeing H, Steffen A, Trichopoulou A, Lagiou P, Kritikou M, Krogh V, Palli D, Sacerdote C, Tumino R, Bueno-de-Mesquita HB, Agudo A, Larranaga N, Molina-Portillo E, Barricarte A, Chirlaque MD, Quiros JR, Stattin P, Haggstrom C, Wareham N, Khaw KT, Schmidt JA, Gunter M, Freisling H, Aune D, Ward H, Riboli E, Key TJ, Travis RC (2017) Tall height and obesity are associated with an increased risk of aggressive prostate cancer: results from the EPIC cohort study. BMC Med 15(1): 115.

Pischon T, Boeing H, Weikert S, Allen N, Key T, Johnsen NF, Tjonneland A, Severinsen MT, Overvad K, Rohrmann S, Kaaks R, Trichopoulou A, Zoi G, Trichopoulos D, Pala V, Palli D, Tumino R, Sacerdote C, Bueno-de-Mesquita HB, May A, Manjer J, Wallstrom P, Stattin P, Hallmans G, Buckland G, Larranaga N, Chirlaque MD, Martinez C, Redondo Cornejo ML, Ardanaz E, Bingham S, Khaw KT, Rinaldi S, Slimani N, Jenab M, Riboli E (2008) Body size and risk of prostate cancer in the European prospective investigation into cancer and nutrition. Cancer Epidemiol Biomarkers Prev 17(11): 3252-3261.

Rider JR, Wilson KM, Sinnott JA, Kelly RS, Mucci LA, Giovannucci EL (2016) Ejaculation frequency and risk of prostate cancer: updated results with an additional decade of follow-up. Eur Urol 70(6): 974-982.

Roddam AW, Allen NE, Appleby P, Key TJ, Endogenous H Prostate Cancer Collaborative G (2008) Endogenous sex hormones and prostate cancer: a collaborative analysis of 18 prospective studies. J Natl Cancer Inst 100(3): 170-183.

Rodriguez C, Patel AV, Mondul AM, Jacobs EJ, Thun MJ, Calle EE (2005) Diabetes and risk of prostate cancer in a prospective cohort of US men. Am J Epidemiol 161(2): 147-152.

Rosenblatt KA, Wicklund KG, Stanford JL (2001) Sexual factors and the risk of prostate cancer. Am J Epidemiol 153(12): 1152-1158.

Sarre S, Maattanen L, Tammela TLJ, Auvinen A, Murtola TJ (2016) Postscreening follow-up of the Finnish Prostate Cancer Screening Trial on putative prostate cancer risk factors: vitamin and mineral use, male pattern baldness, pubertal development and non-steroidal antiinflammatory drug use. Scand J Urol 50(4): 267-273. 
Sudlow C, Gallacher J, Allen N, Beral V, Burton P, Danesh J, Downey P, Elliott P, Green J, Landray M, Liu B, Matthews P, Ong G, Pell J, Silman A, Young A, Sprosen T, Peakman T, Collins R (2015) UK biobank: an open access resource for identifying the causes of a wide range of complex diseases of middle and old age. PLoS Med 12(3): e1001779.

Sutcliffe S, Colditz GA (2013) Prostate cancer: is it time to expand the research focus to early-life exposures? Nat Rev Cancer 13(5): 376.

Teppala S, Shankar A (2010) Association between serum IGF-1 and diabetes among US adults. Diabetes Care 33(10): 2257-2259.

Travis RC, Appleby PN, Martin RM, Holly JM, Albanes D, Black A, Bueno-de-Mesquita HB, Chan JM, Chen C, Chirlaque MD, Cook MB, Deschasaux M, Donovan JL, Ferrucci L, Galan P, Giles GG, Giovannucci EL, Gunter MJ, Habel LA, Hamdy FC, Helzlsouer KJ, Hercberg S, Hoover RN, Janssen JA, Kaaks R, Kubo T, Le Marchand L, Metter EJ, Mikami K, Morris JK, Neal DE, Neuhouser ML, Ozasa K, Palli D, Platz EA, Pollak MN, Price AJ, Roobol M, Schaefer C, Schenk JM, Severi G, Stampfer MJ, Stattin P, Tamakoshi A, Tangen CM, Touvier M, Wald NJ, Weiss NS, Zeigler RG, Key TJ, Allen NE (2016) A meta-analysis of individual participant data reveals an association between circulating levels of IGF-I and prostate cancer risk. Cancer Res 76(8): 2288-2300.

Tsilidis KK, Allen NE, Appleby PN, Rohrmann S, Nothlings U, Arriola L, Gunter MJ, Chajes V, Rinaldi S, Romieu I, Murphy N, Riboli E, Tzoulaki I, Kaaks R, Lukanova A, Boeing H, Pischon T, Dahm CC, Overvad K, Quiros JR, Fonseca-Nunes A, Molina-Montes E, Gavrila Chervase D, Ardanaz E, Khaw KT, Wareham NJ, Roswall N, Tjonneland A, Lagiou P, Trichopoulos D, Trichopoulou A, Palli D, Pala V, Tumino R, Vineis P, Bueno-de-Mesquita HB, Malm J, Orho-Melander M, Johansson M, Stattin P, Travis RC, Key TJ (2015) Diabetes mellitus and risk of prostate cancer in the European Prospective Investigation into Cancer and Nutrition. Int J Cancer 136(2): 372-381.

UK-Biobank (2014) UK Biobank Anthropometry. Available at: http://biob ank.ctsu.ox.ac.uk/crystal/docs/Anthropometry.pdf (accessed on 28 April 2017).
Wallstrom P, Bjartell A, Gullberg B, Olsson H, Wirfalt E (2009) A prospective Swedish study on body size, body composition, diabetes, and prostate cancer risk. Br J Cancer 100(11): 1799-1805.

WCRF/AICR (2014) World Cancer Research Fund International/American Institute for Cancer Research Continuous Update Project Report: Diet, Nutrition, Physical Activity, and Prostate Cancer. Available at: http:// www.wcrf.org/sites/default/files/Prostate-Cancer-SLR-2014.pdf (accessed on 28 April 2017).

Weinstein SJ, Virtamo J, Albanes D (2013) Pigmentation-related phenotypes and risk of prostate cancer. $\mathrm{Br} J$ Cancer 109(3): 747-750.

WHO (2010) International statistical classification of diseases and related health problems. 10th revision. Available at: http://apps.who.int/ classifications/icd10/browse/2010/en (cited 1 April 2016).

Wright JL, Stanford JL (2009) Metformin use and prostate cancer in Caucasian men: results from a population-based case-control study. Cancer Causes Control 20(9): 1617-1622.

Zhou CK, Littman AJ, Levine PH, Hoffman HJ, Cleary SD, White E, Cook MB (2015a) Male pattern baldness in relation to prostate cancer risks: an analysis in the VITamins and Lifestyle (VITAL) Cohort Study. Prostate 75(4): 415-423.

Zhou CK, Pfeiffer RM, Cleary SD, Hoffman HJ, Levine PH, Chu LW, Hsing AW, Cook MB (2015b) Relationship between male pattern baldness and the risk of aggressive prostate cancer: an analysis of the prostate, lung, colorectal, and ovarian cancer screening trial. J Clin Oncol 33(5): 419-U63.

\section{(c) (i)}

This work is licensed under the Creative Commons of this license, visit http://creativecommons.org/licenses/by/4.0/

(C) The Author(s) named above 2017

Supplementary Information accompanies this paper on British Journal of Cancer website (http://www.nature.com/bjc) 